\title{
Clinical Application of Fluorescence Imaging of Liver Cancer Using Indocyanine Green
}

\author{
Norihiro Kokudo Takeaki Ishizawa \\ Hepato-Biliary-Pancreatic Surgery Division, Department of Surgery, \\ Graduate School of Medicine, University of Tokyo, Tokyo, Japan
}

\section{Key Words}

Fluorescence imaging · Hepatocellular carcinoma · Indocyanine green ·

Metastatic liver cancer · Navigation surgery

\begin{abstract}
Recently, fluorescence imaging using indocyanine green (ICG) has been applied to hepatobiliary surgery, not only to visualize the bile ducts, but also to identify liver cancer during surgery. In this technique, ICG is administered intravenously at a dose of $0.5 \mathrm{mg} / \mathrm{kg}$ body weight for routine liver function testing before surgery. Intraoperatively, liver cancer can be readily identified by fluorescence imaging on the liver surface before resection and on the cut surface of the resected specimen. This is achieved by visualizing fluorescence from the area of impaired bile excretion in hepatocellular cancer tissue and in the liver parenchyma surrounding metastatic liver cancers. Liver cancer navigation surgery, first developed in Japan, is also possible, and it represents one of the few fluorescence imaging techniques for cancer that have reached the stage of clinical application; with further developments in basic research, fluorescence imaging is expected to become an indispensable technique for the diagnosis and treatment of liver cancer.

Copyright $\odot 2012$ S. Karger AG, Basel
\end{abstract}

\section{Introduction}

Attempts have long been made to label cancer tissues with fluorescent probes and visualize them in real time during endoscopic examinations or surgical treatment, and

Norihiro Kokudo, MD, PhD

Hepato-Biliary-Pancreatic Surgery Division, Department of Surgery,

Graduate School of Medicine, University of Tokyo

7-3-1 Hongo, Bunkyo-ku, Tokyo 113-8655 (Japan)

Tel. +81 35800 8841, E-Mail KOKUDO-2SU@h.u-tokyo.ac.jp 
fluorescence imaging remains one of the most active key areas of research. However, few techniques have progressed to clinical use, despite the enormous amount of basic research being published. Applications other than the intraoperative imaging of liver cancer with indocyanine green (ICG) [1, 2] are limited to the diagnosis of brain tumors (glioblastomas) [3], bladder cancer [4], and skin tumors [5] and photodynamic therapy using the porphyrin precursor 5-aminolevulinic acid. Herein, we review the historical development and mechanism of liver cancer visualization using ICG fluorescence imaging.

\section{Outline of Surgical Navigation with ICG}

\section{Principle of ICG Fluorescence Imaging}

When protein-bound ICG is exposed to excitation light at 750-810 nm, it emits fluorescence that peaks at about $840 \mathrm{~nm}$ [6]. Because this wavelength is barely absorbed by hemoglobin or water, structures that contain ICG can be visualized through 5-10 mm of tissue by combined use of a camera that is sensitive to infrared light and an appropriate filter (the ICG fluorescence imaging technique). These properties are optimal for performing contrast studies of vascular structures, and in the early 1990s, ICG fluorescence imaging was applied clinically to fundus angiography in the field of ophthalmology [7]. However, probably because ICG was widely adopted as a reagent for measuring cardiac output or measuring liver function, little attention was paid to ICG's fluorescence properties in the fields of thoracic surgery or general surgery, and there have been few attempts to apply it to surgical navigation.

\section{Application to Surgical Navigation}

At the beginning of the 21st century, several surgical navigation techniques utilizing the fluorescence properties of ICG were developed in rapid succession. Coronary arteriography during cardiac bypass surgery using ICG fluorescence imaging was developed first [8]. Then, the fluorescence properties of ICG were applied to lymphangiography [9] and the identification of sentinel nodes during breast cancer resections [10]; at present, its application has expanded to cholangiography during hepatobiliary surgery [11-13] and to evaluations of the blood flow after the clipping of cerebral arterial aneurysms [14].

One of these applications, the identification of breast cancer sentinel nodes, was the first application of ICG fluorescence imaging to the surgical treatment of cancer. However, this method visualizes lymphatic drainage routes from the cancer tissue and allows searches for lymph nodes, but does not allow visualization of the ICG that has been taken up specifically by cancer tissues. The nature of fluorescence imaging of hepatocellular carcinoma (HCC) [1, 2], which was first described in Japan in 2009, is very different from that of other techniques: it allows visualization of the HCC itself by fluorescence imaging of the ICG that is taken up and remains in the cancer tissues as a result of biliary excretion disorders. This gives high lesion-to-background contrast [median (range), 2.6 (0.6-16.6) fold, unpublished data] for well- or moderately differentiated HCC, which is higher than that for other kinds of cancer tissues (up to 2 fold) $[15,16]$.

Liver cancer imaging by the ICG fluorescence imaging technique was patented by a group at the University of Rochester (US) in 2008 (WO 2008/043101 A2), although there have been no detailed reports in respect of such liver cancer imaging, except for a recent article on the application of this technique to robotic surgery for identification of renal cancer [17]. Based on a reading of the patent details, their method probably utilizes the difference in hemodynamics between liver cancer tissue and the surrounding liver tissue, 
which is different from the Japanese technique that is based on impaired biliary excretion in HCC tissues.

\section{Development of Liver Cancer Imaging Using ICG}

\section{Discovery of Fluorescence Imaging of Liver Cancer}

The authors originally developed a cholangiographic method based on ICG fluorescence imaging $[13,14]$. During the process of that development, we noticed that tumors on the liver surface emitted their own fluorescence even before ICG was injected into the bile duct for cholangiography. All patients in our department are administered ICG intravenously to measure the ICG retention rate at $15 \mathrm{~min}$ as a routine preoperative liver function test $[18,19]$. Thus, it was assumed that the intraoperative visualization of liver cancer by ICG fluorescence imaging must have been because the ICG that had been injected intravenously prior to the surgery had remained in the liver cancer or surrounding liver tissues. A prospective clinical study was immediately initiated, and the follow-up results for 63 HCCs (37 cases) and 28 metastatic liver nodules from colorectal cancer (12 cases) were reported in 2009 [1]. At the same time, Gotoh et al. reported the results of ICG fluorescence imaging of 14 HCCs (10 cases) that included 4 nodules first discovered by this technique [2]. Yokoyama et al. recently added a report on the possibility of applying ICG fluorescence imaging to the intraoperative diagnosis of micrometastases of pancreatic cancer [20].

\section{Mechanism of Fluorescence Imaging of Liver Cancer}

In the authors' study, well-differentiated HCC tissue exhibited intense fluorescence on the cut surface of surgically resected liver specimens, whereas for poorly differentiated HCC and metastases of colorectal cancer, the cancer tissue itself was not visualized, but the surrounding noncancerous tissues produced a ring of fluorescence (fig. 1) [21]. Moreover, observations made with a fluorescence microscope confirmed the presence of ICG fluorescence in the cytoplasm and pseudoglands in well-differentiated HCCs; fluorescence was also seen in nontumorous liver tissues compressed by the tumor in cases of poorly differentiated HCCs and colorectal cancer metastases. Based on the above results, it is suggested that well-differentiated HCC tissues take up ICG, but the biliary excretion of ICG is impaired because of morphological or functional abnormalities. In contrast, poorly differentiated HCCs and metastatic liver cancers do not take up ICG, but they can be visualized by rings of flourescence resulting from impaired bile excretion in the surrounding nontumorous tissues compressed by the tumors. Consequently, well-differentiated HCCs, poorly differentiated HCCs, and metastatic liver cancers can all be visualized by ICG fluorescent imaging.

The existence of impaired bile excretion in HCC and in noncancerous tissues surrounding the cancer had been suggested by the results of magnetic resonance imaging with a bile-excreted contrast medium [22]. Moreover, Kitao et al. demonstrated reduced expression of organic anion transporting polypeptide 8 , which is involved in the cellular uptake of contrast media, in poorly differentiated HCC compared with that in well-differentiated HCC [23], and the differences between the enhancement patterns of well- and poorly differentiated HCC by ICG fluorescence imaging may also be attributable to a similar mechanism. In addition, through approaches utilizing immunostaining and gene expression analyses, it is hoped that progress will be made in elucidating the mechanism of liver cancer imaging by ICG fluorescence imaging using a recently developed fluorescence endomicroscopy system [24] and in making real-time observations of the pharmacokinetics of ICG in liver cancers and the surrounding tissues. 
Fig. 1. Fluorescence patterns of liver cancers on cut surfaces (left) and their gross appearance (right) (from [21]). a Total fluorescence type (welldifferentiated HCC, $7 \mathrm{~mm}$ in diameter). b Partial fluorescence type (moderately differentiated HCC, $35 \mathrm{~mm}$ in diameter). c Rim fluorescence type (poorly differentiated HCC, $30 \mathrm{~mm}$ in diameter). d Rim fluorescence type (metastasis of colorectal cancer, $25 \mathrm{~mm}$ in diameter).
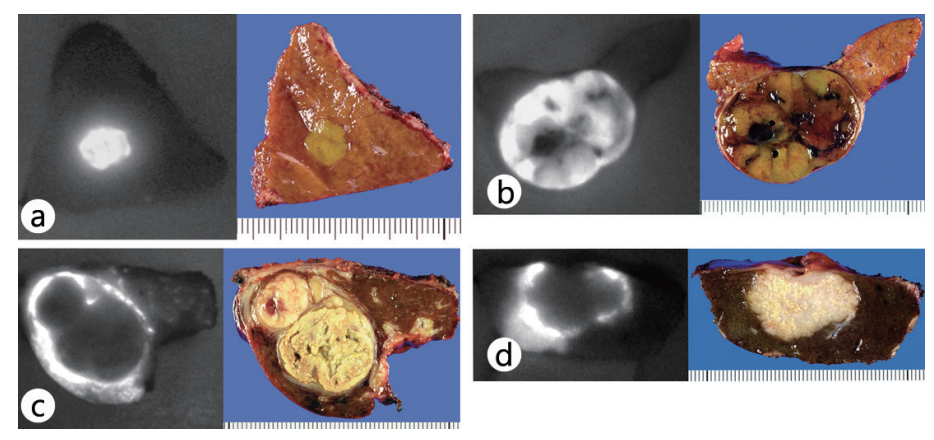

Fig. 2. ICG fluorescence imaging on liver surfaces (left) and their gross appearances (right) (from [21]). a Fluorescence imaging prior to liver resection enabled visualization of the metastasis of colorectal cancer that was palpable but grossly unidentifiable (arrow), as well as the other two lesions visible on the liver surface. b Fluorescence imaging of the resected specimen. The arrow indicates a grossly unidentifiable tumor that was located 0.8 $\mathrm{cm}$ below the liver surface.
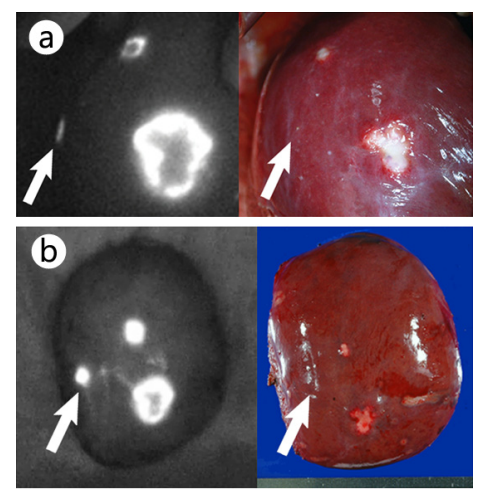

\section{Techniques and Precautions for ICG Fluorescence Imaging of Liver Cancer}

The technique of ICG fluorescence imaging of liver cancer is very simple: once ICG 0.5 $\mathrm{mg} / \mathrm{kg}$ body weight) is administered within 14 days prior to surgery, we can obtain fluorescence images of liver cancers intraoperatively by simply placing the camera imaging head of commercially available fluorescence imaging systems on the liver surface or on surgical specimens (fig. 2) [1, 21]. In patients with bile duct infiltrations of liver cancer, fluorescence imaging is also possible for biliary congested regions on the liver surface, which may be helpful for surgeons in determining the hepatic transection line [25].

It should be noted that if ICG is injected intravenously on the day before surgery, washout from the noncancerous liver tissue is inadequate and there may be many false-positive nodules; the poorer the liver function is, the more marked this tendency is. Further studies are needed to determine the optimal interval between ICG injection and surgery on the basis of the patient's liver function. Moreover, this technique does not use an antigen-antibody reaction that is specific for cancer, it just allows visualization of the areas of impaired bile excretion in or around the tumor tissues; consequently, benign lesions such as regenerating nodules or bile duct proliferation may also exhibit fluorescence. Previous reports have indicated that $40-50 \%$ of the lesions newly discovered by fluorescence imaging of resected HCC specimens were noncancerous lesions [1,2]. Thus, the principal role of this method lies in intraoperatively searching for small liver cancers near the liver surface that were detected by preoperative imaging studies; any additional resection of fluorescent areas that are newly discovered intraoperatively by the ICG fluorescence imaging technique should be considered only after they have been adequately confirmed by inspection and palpation and/or by an intraoperative ultrasound examination. 


\section{Problems with the ICG Fluorescence Imaging Technique and Future Prospects}

\section{Problems with ICG Fluorescence Liver Cancer Imaging}

ICG fluorescence imaging has the advantage of being a phenomenon that is easy to understand - there is fluorescence where there is bile - and of being simple and highly sensitive; however, because it identifies liver cancer by visualizing impaired biliary excretion of ICG, it may be less specific than pre-clinical fluorescence imaging techniques using a cancer-specific antibody. Moreover, since the tissue penetration of the fluorescence emitted by ICG is only about $5-10 \mathrm{~mm}$, it is impossible to visualize tumors located deep within the liver. Although ICG fluorescence imaging is already a sufficiently practical technique for hepatic surgeons, it is important to understand the characteristics of the technique and to utilize it in a complementary manner with inspection and palpation or an intraoperative ultrasound examination.

\section{New Techniques Likely To Be Developed for the Clinical Setting}

If the development progresses satisfactorily of a novel fluorescent probe [26] that is excreted in the bile by the same route as ICG and whose tissue penetration is superior to that of ICG, then the sensitivity of imaging for liver cancer with this technique may improve. Moreover, if ICG can be modified with a cancer-specific antibody or other tracer [27], it might not only be possible to use it for surgical navigation, but it may evolve into a new preoperative imaging technique that simultaneously allows tumor diagnosis and localization, evaluation of the segmental liver function. In particular, photodynamic therapy for cancer using a compound consisting of a tumor-specific antibody and a photosensitive substance has recently attracted much attention [28]. Since ICG is also a photosensitive substance, a novel photodynamic therapy that takes advantage of this property of ICG and its accumulation in liver cancer may be possible.

It is also essential to improve the performance of imaging devices. It is already possible to simultaneously acquire color images and fluorescence images $[29,30]$ and to add an infrared observation system to laparoscopic surgery systems [13, 31, 32] and to a robotic surgery system [17]. If it becomes possible to visualize ICG fluorescence that is emitted from deeper regions, this would contribute to further improvement of the accuracy of resection for liver cancer.

\section{Conclusions}

ICG fluorescence imaging is quite a simple technique that enables highly sensitive identification of liver cancers in real time during surgery. This technique is also valuable from the standpoint of being one of the few fluorescence imaging techniques for cancer that have reached clinical application. Further basic research should result in innovative techniques in the near future, and this technique may evolve to play a major clinical role in the diagnosis and treatment of liver cancer.

\section{Acknowledgments}

This work was supported by grants from the Takeda Science Foundation, the Kanae Foundation for the Promotion of Medical Science, and the Ministry of Education, Culture, Sports, Science and Technology of Japan (No. 23689060 and 23249067). 


\section{References}

1 Ishizawa T, Fukushima N, Shibahara J, et al: Real-time identification of liver cancers by using indocyanine green fluorescent imaging. Cancer 2009;115:2491-2504.

2 Gotoh K, Yamada T, Ishikawa 0, et al: A novel image-guided surgery of hepatocellular carcinoma by indocyanine green fluorescence imaging navigation. J Surg Oncol 2009;100:75-79.

3 Stummer W, Stocker S, Wagner S, et al: Intraoperative detection of malignant gliomas by 5-aminolevulinic acid-induced porphyrin fluorescence. Neurosurgery 1998;42:518-525.

4 Kriegmair M, Stepp H, Steinbach P, et al: Fluorescence cystoscopy following intravesical instillation of 5-aminolevulinic acid: a new procedure with high sensitivity for detection of hardly visible urothelial neoplasias. Urol Int 1995;55:190-196.

5 Morton CA, Brown SB, Collins S, et al: Guidelines for topical photodynamic therapy: report of a workshop of the British Photodermatology Group. Br J Dermatol 2002;146:552-567.

6 Landsman ML, Kwant G, Mook GA, et al: Light-absorbing properties, stability, and spectral stabilization of indocyanine green. J Appl Physiol 1976;40:575-583.

7 Guyer DR, Puliafito CA, Monés JM, et al: Digital indocyanine-green angiography in chorioretinal disorders. Ophthalmology 1992;99:287-291.

8 Rubens FD, Ruel M, Fremes SE: A new and simplified method for coronary and graft imaging during CABG. Heart Surg Forum 2002;5:141-144.

9 Ogata F, Azuma R, Kikuchi M, et al: Novel lymphography using indocyanine green dye for near-infrared fluorescence labeling. Ann Plast Surg 2007;58:652-655.

10 Kitai T, Inomoto T, Miwa M, et al: Fluorescence navigation with indocyanine green for detecting sentinel lymph nodes in breast cancer. Breast Cancer 2005;12:211-215.

11 Mitsuhashi N, Kimura F, Shimizu H, et al: Usefulness of intraoperative fluorescence imaging to evaluate local anatomy in hepatobiliary surgery. J Hepatobiliary Pancreat Surg 2008;15:508-514.

12 Ishizawa T, Tamura S, Masuda K, et al: Intraoperative fluorescent cholangiography using indocyanine green: a biliary road map for safe surgery. J Am Coll Surg 2009;208:e1-e4.

13 Ishizawa T, Bandai Y, Ijichi M, et al: Fluorescent cholangiography illuminating the biliary tree during laparoscopic cholecystectomy. Br J Surg 2010;97:1369-1377.

14 Raabe A, Nakaji P, Beck J, et al: Prospective evaluation of surgical microscope-integrated intraoperative near-infrared indocyanine green videoangiography during aneurysm surgery. J Neurosurg 2005; 103:982-989.

15 Thayer D, Unlu MB, Lin Y, et al: Dual-contrast dynamic MRI-DOT for small animal imaging. Technol Cancer Res Treat 2010;9:61-70.

16 Kim TH, Mount CW, Dulken BW, et al: Filamentous, mixed micelles of triblock copolymers enhance tumor localization of indocyanine green in a murine xenograft model. Mol Pharm 2012;9:135-143.

17 Tobis S, Knopf J, Silvers C, et al: Near infrared fluorescence imaging with robotic assisted laparoscopic partial nephrectomy: initial clinical experience for renal cortical tumors. J Urol 2011;186:47-52.

18 Makuuchi M, Kosuge T, Takayama T, et al: Surgery for small liver cancers. Semin Surg Oncol 1993;9:298304.

19 Ishizawa T, Hasegawa K, Aoki T, et al: Neither multiple tumors nor portal hypertension are surgical contraindications for hepatocellular carcinoma. Gastroenterology 2008;134:1908-1916.

20 Yokoyama N, Otani T, Hashidate H, et al: Real-time detection of hepatic micrometastases from pancreatic cancer by intraoperative fluorescence imaging: Preliminary results of a prospective study. Cancer 2011; (in press).

21 Ishizawa T, Harada N, Muraoka A, et al: Scientific basis and clinical application of ICG fluorescence imaging: hepatobiliary cancer. Open Surg Oncol J 2010;2:31-36.

22 Chung JJ, Kim MJ, Kim KW: Mangafodipir trisodium-enhanced MRI for the detection and characterization of focal hepatic lesions: is delayed imaging useful? J Magn Reson Imaging 2006;23:706-711.

23 Kitao A, Zen Y, Matsui O, et al: Hepatocellular carcinoma: signal intensity at gadoxetic acid-enhanced MR Imaging-correlation with molecular transporters and histopathologic features. Radiology 2010;256:817826.

24 Goetz M, Deris I, Vieth M, et al: Near-infrared confocal imaging during mini-laparoscopy: a novel rigid endomicroscope with increased imaging plane depth. J Hepatol 2010;53:84-90.

25 Harada N, Ishizawa T, Muraoka A, et al: Fluorescence navigation hepatectomy by visualization of localized cholestasis from bile duct tumor infiltration. J Am Coll Surg 2010;210:e2-e6.

26 Figueiredo JL, Siegel C, Nahrendorf M, et al: Intraoperative near-infrared fluorescent cholangiography (NIRFC) in mouse models of bile duct injury. World J Surg 2010;34:336-343.

27 Ogawa M, Kosaka N, Choyke PL, et al: In vivo molecular imaging of cancer with a quenching near-infrared fluorescent probe using conjugates of monoclonal antibodies and indocyanine green. Cancer Res 2009;69:1268-1272.

28 Mitsunaga M, Ogawa M, Kosaka N, et al: Cancer cell-selective in vivo near infrared photoimmunotherapy targeting specific membrane molecules. Nat Med 2011;17:1685-1691.

29 Kawaguchi Y, Ishizawa T, Masuda K, et al: Hepatobiliary surgery guided by a novel fluorescent imaging technique for visualizing hepatic arteries, bile ducts, and liver cancers on color images. J Am Coll Surg 2011;212:e33-e39.

30 Matsui A, Winer JH, Laurence RG, et al: Predicting the survival of experimental ischaemic small bowel using intraoperative near-infrared fluorescence angiography. Br J Surg 2011;98:1725-1734. 
31 Ishizawa T, Bandai Y, Harada N, et al: Indocyanine green-fluorescent imaging of hepatocellular carcinoma during laparoscopic hepatectomy: an initial experience. Asian J Endosc Surg 2010;3:42-45.

32 Ishizawa T, Zuker N, Kokudo N, et al: Positive and negative staining of hepatic segments by fluorescent imaging technique during laparoscopic hepatectomy. Arch Surg 2012; (in press). 